\title{
Why does a point of care guided transfusion algorithm not improve blood loss and transfusion practice in patients undergoing high-risk cardiac surgery? A prospective randomized controlled pilot study
}

F. Lehmann ${ }^{1 *}$ D, J. Rau ${ }^{2}$, B. Malcolm³ ${ }^{3}$ M. Sander ${ }^{4}$, C. von Heymann ${ }^{5}$, T. Moormann ${ }^{6}$, T. Geyer ${ }^{1}$, F. Balzer ${ }^{1}$,

K. D. Wernecke ${ }^{7}$ and L. Kaufner ${ }^{1}$

\begin{abstract}
Background: Adult cardiac surgery is often complicated by elevated blood losses that account for elevated transfusion requirements. Perioperative bleeding and transfusion of blood products are major risk factors for morbidity and mortality. Timely diagnostic and goal-directed therapies aim at the reduction of bleeding and need for allogeneic transfusions.
\end{abstract}

Methods: Single-centre, prospective, randomized trial assessing blood loss and transfusion requirements of 26 adult patients undergoing elective cardiac surgery at high risk for perioperative bleeding. Primary endpoint was blood loss at $24 \mathrm{~h}$ postoperatively. Random assignment to intra- and postoperative haemostatic management following either an algorithm based on conventional coagulation assays (conventional group: platelet count, aPTT, PT, fibrinogen) or based on point-of-care (PoC-group) monitoring, i.e. activated rotational thromboelastometry $\left(\right.$ ROTEM $\left.^{\oplus}\right)$ combined with multiple aggregometry (Multiplate ${ }^{\oplus}$ ). Differences between groups were analysed using nonparametric tests for independent samples.

Results: The study was terminated after interim analysis $(n=26)$. Chest tube drainage volume was $360 \mathrm{ml}$ (IQR 229$599 \mathrm{ml}$ ) in the conventional group, and $380 \mathrm{ml}(\mathrm{IQR} 310-590 \mathrm{ml})$ in the PoC-group ( $p=0.767)$ after $24 \mathrm{~h}$. Basic patient characteristics, results of PoC coagulation assays, and transfusion requirements of red blood cells and fresh frozen plasma did not differ between groups. Coagulation results were comparable. Platelets were transfused in the PoC group only.

Conclusion: Blood loss via chest tube drainage and transfusion amounts were not different comparing PoC-and central lab-driven transfusion algorithms in subjects that underwent high-risk cardiac surgery. Routine PoC coagulation diagnostics do not seem to be beneficial when actual blood loss is low. High risk procedures might not suffice as a sole risk factor for increased blood loss.

Trial registration: NCT01402739, Date of registration July 26, 2011.

Keywords: Algorithms, Blood coagulation, Blood transfusion, Point-of-care systems, Thoracic surgery

\footnotetext{
* Correspondence: ferdinand.lehmann@charite.de

'Department of Anaesthesiology and Intensive Care Medicine, Charité -

Universitätsmedizin Berlin, Campus Virchow-Klinikum and Campus Charité

Mitte, Berlin, Germany

Full list of author information is available at the end of the article
}

(c) The Author(s). 2019 Open Access This article is distributed under the terms of the Creative Commons Attribution 4.0 International License (http://creativecommons.org/licenses/by/4.0/), which permits unrestricted use, distribution, and reproduction in any medium, provided you give appropriate credit to the original author(s) and the source, provide a link to the Creative Commons license, and indicate if changes were made. The Creative Commons Public Domain Dedication waiver (http://creativecommons.org/publicdomain/zero/1.0/) applies to the data made available in this article, unless otherwise stated. 


\section{Background}

Adult cardiac surgery patients are at increased risk of perioperative bleeding, either due to concomitant medication [1,2] or the type of surgery itself [3]. Increased blood loss is associated with increased need for allogeneic blood transfusions [4] and poorer outcome [5, 6]. Minimising blood loss and bleeding is the second pillar of patient blood management [7, 8], a multimodal concept that is encouraged by the World Health Organization [9]. Haemostasis management is one aspect to minimize blood loss [7]. For red blood cells, algorithm-based transfusion is considered safe and associated with comparably low transfusion rates $[6,10,11]$.

Enforceable transfusion algorithms are part of the recommendation on the management of blood resources of the 2011 Update to The Society of Thoracic Surgeons and the Society of Cardiovascular Anesthesiologists Blood Conservation Clinical Practice Guidelines [12], and the recent guideline of the European Association of Cardiothoracic Anaesthesiology (EACTA) in conjunction with the European Association of Cardio-thoracic Surgeons (EACTS) [13]. The use of transfusion algorithms itself has been shown to significantly reduce patients' exposure to blood products in cardiac surgery [13-16].

Perioperative monitoring of coagulation may help to identify the underlying causes for bleeding and enable specific treatment. Point-of-care (PoC) coagulation monitoring is widely used and has been recommended in various guidelines [12, 13, 17-19].

As reviewed earlier, several studies focused on PoCguided transfusion algorithms compared to algorithms based on conventional coagulation testing, clinician discretion or "standard of care" in cardiac surgery [14, 20]. Few were randomized controlled trials [15, 21-26]. Only two studies applied explicit transfusion algorithms in both, the PoC- and conventional coagulation testsguided group [23, 24]. Of those, one study applied the transfusion protocols inside the operation theatre only [23], although a relevant number of bleeding episodes and transfusions occurs postoperatively [6, 24].

Any type of algorithm allows defining a goal-directed transfusion strategy [16]. Furthermore, the implementation of an algorithm itself has been shown to improve standardisation and outcome [27]. The question remains, whether the superior results of PoC-guided transfusion algorithms [20] are reproducible in a setting of restrictive and explicit transfusion algorithms both in PoC- and conventional coagulation assay-guided protocols.

Therefore, we conducted a prospective randomized trial in cardiac surgical procedures at high risk for bleeding that compared transfusion algorithms either guided by $\mathrm{PoC}$ (activated rotational thromboelastometry, and multiple electrode aggregometry (MEA)) or standard coagulation assays. We assumed that $\mathrm{PoC}$ coagulation monitoring is superior to standard coagulation measurements due to shorter turnaround times of test results enabling a faster and more specific treatment with haemostatic products. Our hypothesis was a reduction of postoperative blood loss via chest tube drainage and transfusion needs in patients undergoing high-risk cardiac surgery treated following a PoC-driven transfusion protocol compared to a central lab-guided protocol.

\section{Methods}

This single centre trial has a prospective, randomized parallel-group design with two study arms $(n=100$, Fig. 2). First study arm focused on patients undergoing high risk cardiac surgery ( $n=50$, Fig. 2 , data shown). Second study arm investigated patients with dual platelet inhibition undergoing cardiac surgery $(n=50$, Fig. 2, data not shown). The study was conducted at the Department of Anaesthesiology and Intensive Care Medicine of Charité - University Medicine Berlin, Berlin, Germany in adherence to the latest version of the declaration of Helsinki, approved by the local Ethics Board (Ethics Committee of Charité - University Medicine Berlin (EA1/263/10). The trial is registered with clinicaltrials.gov (identifier NCT01402739) and adheres to CONSORT guidelines. All patients gave written informed consent prior to entering the study.

For the first study arm, patients were eligible for participation if aged 18-80 yrs. and scheduled for cardiac surgery using cardio-pulmonary bypass $(\mathrm{CPB})$ for either a combined CABG/valve procedure, a double or triple valve procedure or a redo surgery, defining patients as at high risk for bleeding and transfusion [3]. Key exclusion criteria were hereditary or acquired defects in haemostasis (see Appendix for all exclusion criteria) and surgical procedures not regarded as of high risk of bleeding. After enrolment, participants were assigned to the PoC or the conventional group by simple, stratified envelope randomization with allocation concealment aiming at 1:1 assignment. The stratum was risk for bleeding, and the person conducting randomization was neither the person enrolling the patients, nor one of the treating physicians. The primary outcome parameter was the chest tube drainage volume after $24 \mathrm{~h}$. Secondary outcome parameters included the course of chest tube drainage at 6,12 and $24 \mathrm{~h}$ postoperatively, the need of allogeneic blood transfusions in the first $24 \mathrm{~h}$, the course of conventional coagulation parameters, the duration of mechanical ventilation, and the incidence of renal replacement therapy.

\section{Perioperative management}

Anaesthetic, surgical, $\mathrm{CPB}$ and postoperative intensive care management were standardised for each patient. Changes to local standard management applied to 
coagulation monitoring and transfusion management according to the study protocol only. Intraoperative blood losses were salvaged and washed before retransfusion to avoid heparin effects.

Anaesthetic management was conducted following local standard operating procedures for cardiac surgery patients. Routine monitoring included continuous arterial blood pressure and central venous pressure monitoring, 5-lead ECG, pulse-oximetry and BIS monitoring. General anaesthesia was induced with etomidate $0.2-0.3$ $\mathrm{mg} / \mathrm{kg}$ body weight $(\mathrm{kgBW})$, sufentanil $0.2-0.4 \mu \mathrm{g} / \mathrm{kgBW}$ and cis-atracurium $0.1-0.15 \mathrm{mg} / \mathrm{kgBW}$. Anaesthesia was maintained with sufentanil $0.5-1.0 \mu \mathrm{g} / \mathrm{kgBW} / \mathrm{h}$ and sevoflurane 0.7-1 MAC. During normothermic CPB in addition to sufentanil, propofol $2-4 \mathrm{mg} / \mathrm{kgBW} / \mathrm{h}$ was infused to ensure sufficient anaesthetic depth. Tranexamic acid was dosed according to the BART protocol in all patients [28].

Blood samples for coagulation monitoring were taken from the arterial line prior to induction, after the start of $\mathrm{CPB}$, after aortic declamping and administration of protamine as well as at $1 \mathrm{~h}, 6 \mathrm{~h}, 24 \mathrm{~h}$ and $48 \mathrm{~h}$ postoperatively.

\section{Transfusion protocols}

Transfusion protocols were used from induction of general anaesthesia until $24 \mathrm{~h}$ postoperatively:

Packed red blood cells (RBC). For both groups, a previously described protocol used safely in moderaterisk patients undergoing cardiac surgery was applied [10]. Briefly, a haemoglobin of $\leq 6 \mathrm{~g} / \mathrm{dl}$ led to a transfusion of one unit RBC. For haemoglobin values $6-8 \mathrm{~g} / \mathrm{dl}$, transfusion was acceptable, but not mandatory. For haemoglobin values of 8-10 g/dl, transfusion of one unit $\mathrm{RBC}$ was acceptable if at least one of the following was present: $\mathrm{ScvO} 2<70 \%$ if arterial $\mathrm{SpO} 2>90 \%$, cardiac index $<2.5$ refractory towards inotropes or mechanical support, or symptoms of end-organ ischemia.

Fresh frozen plasma (FFP), platelet concentrates, and fibrinogen concentrate. For both groups, transfusion of these blood products was allowed either before chest closure in case of severe diffuse bleeding delaying chest closure as assessed by the cardiac surgeon and anaesthesiologist, or in case of blood loss exceeding 1.5 $\mathrm{ml} / \mathrm{kgBW} /$ hour in two consecutive hours or $4 \mathrm{ml} /$ $\mathrm{kgBW} /$ hour for at least $30 \mathrm{~min}$ postoperatively.

In the conventional group, $10 \mathrm{ml} / \mathrm{kgBW}$ FFP was transfused if INR $>1.5$. A platelet count of $\leq 100 / \mathrm{nl}$ led to a transfusion of one apheresis platelet concentrate unit. If fibrinogen was $\leq 150 \mathrm{mg} / \mathrm{dl}, 2 \mathrm{~g}$ of fibrinogen concentrate were administered. Twenty-five mg of Protamine could be administered in patients whose ACT was $>10 \%$ or whose aPTT was $>20 \%$ above the upper reference range $(120 \mathrm{~s}$. and 26-40 s., respectively). In cases of persistent bleeding despite surgical haemostasis or after prolonged CPB time, or pre-operative antiplatelet medication, platelets and/or FFP were acceptable at the discretion of the attending anaesthesiologist. In cases of normal conventional coagulation parameters and a suspected platelet disorder desmopressin $0.3 \mu \mathrm{g} / \mathrm{kgBW}$ was considered.

In the $\mathrm{PoC}$ group, parallel measurements using the activated rotational thromboelastometry (ROTEM ${ }^{\mathrm{sm}}$, TEM International $\mathrm{GmbH}$, Munich, Germany) and MEA (Multiplate ${ }^{\circ}$ Analyzer, Roche Deutschland, Mannheim, Germany) were performed. Activated rotational thromboelastometry measures clot-building, clot firmness, clot lysis and their dynamics in recalcified citrated whole blood [29]. MEA reflects platelet aggregation ability in anticoagulated whole blood, being able to differentiate platelet inhibition by acetylsalicylic acid, thienopyridines and GPIIbIIIa inhibitors [30]. The haemostatic management algorithms are given in Fig. 1. Additionally, in cases of persistent bleeding despite normal PoC parameters desmopressin $0.3 \mu \mathrm{g} / \mathrm{kgBW}$ was considered if surgical bleeding was denied.

For both groups, the application of the prothrombin complex concentrate, antithrombin, recombinant factor VIIa, factors VIII, IX and XIII was acceptable in case of diagnosed deficiencies or therapy-refractory bleeding.

\section{Coagulation testing}

In both groups, blood samples for conventional coagulation assays and point-of-care assays were collected and analysed simultaneously. Results of $\mathrm{PoC}$ testing were available to the attending physicians in the PoC group only but recorded for study purposes for both groups. Results of the conventional coagulation tests were automatically displayed in the electronic patient chart but were irrelevant for PoC group patients both due to later availability caused by longer turnaround times and a transfusion algorithm independent of conventional coagulation tests.

\section{Statistics}

For the first study arm (Fig. 2) we hypothesized that 250 $\mathrm{ml}$ difference in chest tube drainage volume would be clinically relevant. The sample size calculation performed by CRO SOSTANA GmbH, Berlin, Germany, yielded $n=24$ per group to detect this difference over $24 \mathrm{~h}$ with a type one error of $\alpha=5 \%$ (two-sided) and a power of $80 \%$. The calculation was based on the chest tube drainage volume in the control group of a published study for a common standard deviation of 281.69 $\mathrm{ml}$, investigating different cardiac surgical procedures resembling rather a high risk of bleeding population than patients undergoing one specific surgical procedure [30]. Therefore, a group size of $n=25$ was chosen as appropriate. 
A

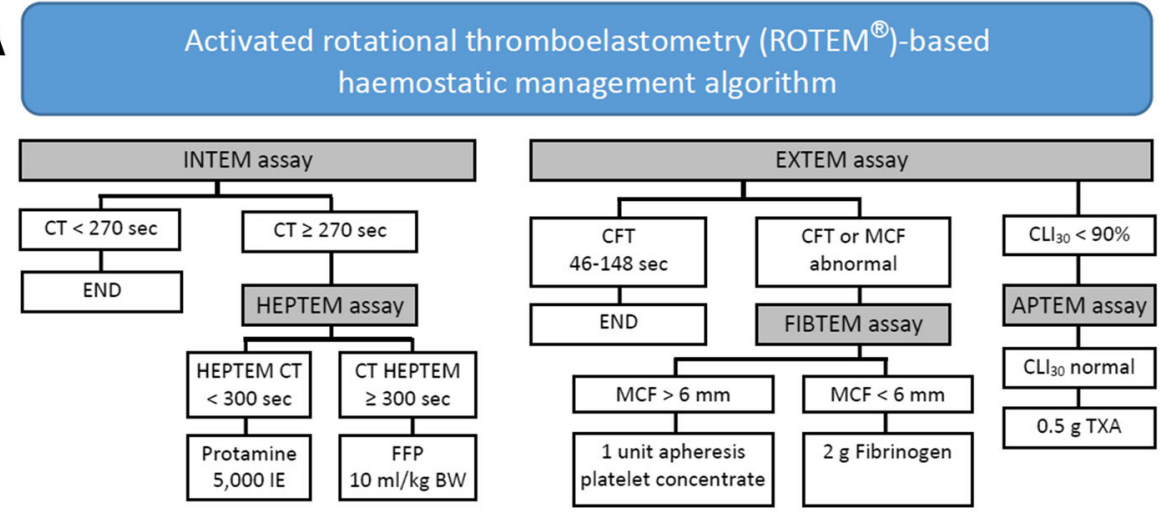

B

Multiple electrode aggregometry (MEA)-based haemostatic management algorithm

\begin{tabular}{|l|}
\hline \multicolumn{1}{|c|}{ TRAP assay } \\
\hline $\mathrm{AU}>65.8:$ \\
no action \\
$\mathrm{AU}$ 37.6-65.8: \\
$>\quad$ Desmopressin $0.3 \mu \mathrm{g} / \mathrm{kgBW}$ \\
$\mathrm{AU}<37.6:$ \\
$>\quad 1$ unit apheresis platelet \\
concentrate
\end{tabular}
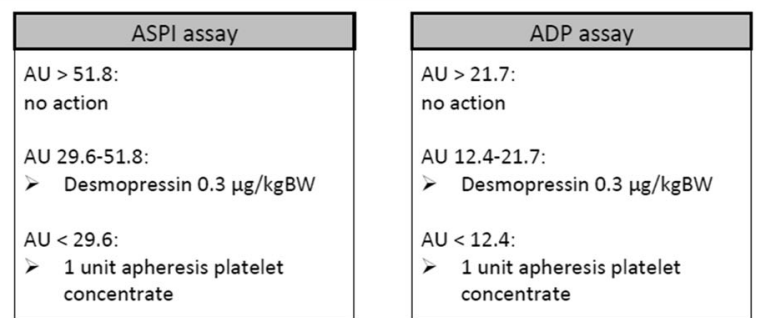

Fig. 1 PoC therapy algorithms for the PoC group in case of blood loss exceeding $1.5 \mathrm{ml} / \mathrm{kg}$ body weight (kgBW)/hour in two consecutive hours or $4 \mathrm{ml} / \mathrm{kgBW} /$ hour for at least $30 \mathrm{~min}$, or bleeding delaying chest closure intraoperatively. a Activated rotational thromboelastometry (ROTEM ${ }^{\oplus}$-based haemostatic management algorithm. b Multiple electrode aggregometry (MEA)-based haemostatic management algorithm

An interim analysis of the primary outcome parameter blood loss in the first study arm was performed by CRO SOSTANA GmbH, Berlin, Germany, after $50 \%$ of the planned number of patients were enrolled in each group just for the purpose to validate the sample size calculation of the study arm, but not for statistical testing.

Results are given as median (interquartile ranges (IQR)). Differences between groups were analysed using the nonparametric Mann-Whitney U test or Chi-square test (categorical data) for two independent samples. Multivariate nonparametric analysis of longitudinal data in a two-factorial design (1st factor: groups, 2nd factor: repetitions in time) was used to analyse time courses. A two-tailed $p$-value $<0.05$ was considered significant. Tests for secondary outcome parameters were conducted as exploratory data analysis. Therefore, no adjustments for multiple testing were applied. Calculations were performed with IBM SPSS Statistics for Windows, Versions 22.0 and 23.00, Armonk, NY: IBM Corp., or The R Project for Statistical Computing, Version 3.0.2 (2013-09-25). Propensity score matching was based on genetic matching algorithm with automated balance optimization [31] and applied with the $\mathrm{R}$ package "Matching" [32].

\section{Results}

Enrolment of both study arms started August 23rd 2011. In total, 1306 patients were assessed for eligibility until March 20th 2014. Twenty-six patients were enrolled and randomized in the first arm that is described here only (results of second study arm will be described elsewhere). One patient had to be excluded pre-operatively due to technical reasons. Fourteen patients in the conventional group and 11 patients in the $\mathrm{PoC}$ group were analysed (Fig. 2). The first study arm was terminated early after the planned interim analysis. The revised sample size calculation for the first study arm based on the chest tube drainage volume in our patient population yielded 13,131 patients had to be recruited per group. This was considered not feasible in a single centre study.

The basic patient and surgery characteristics are given in Table 1. There were no significant differences between groups. The primary endpoint, chest tube drainage volume at $24 \mathrm{~h}$ postoperatively, did not differ $(p=0.767)$. Chest tube drainage volume of the first $24 \mathrm{~h}$ postoperatively is given in Fig. 3, revealing no differences between groups over the time. Transfusion rates are given in Table 2, revealing transfusions of platelets in the PoC 


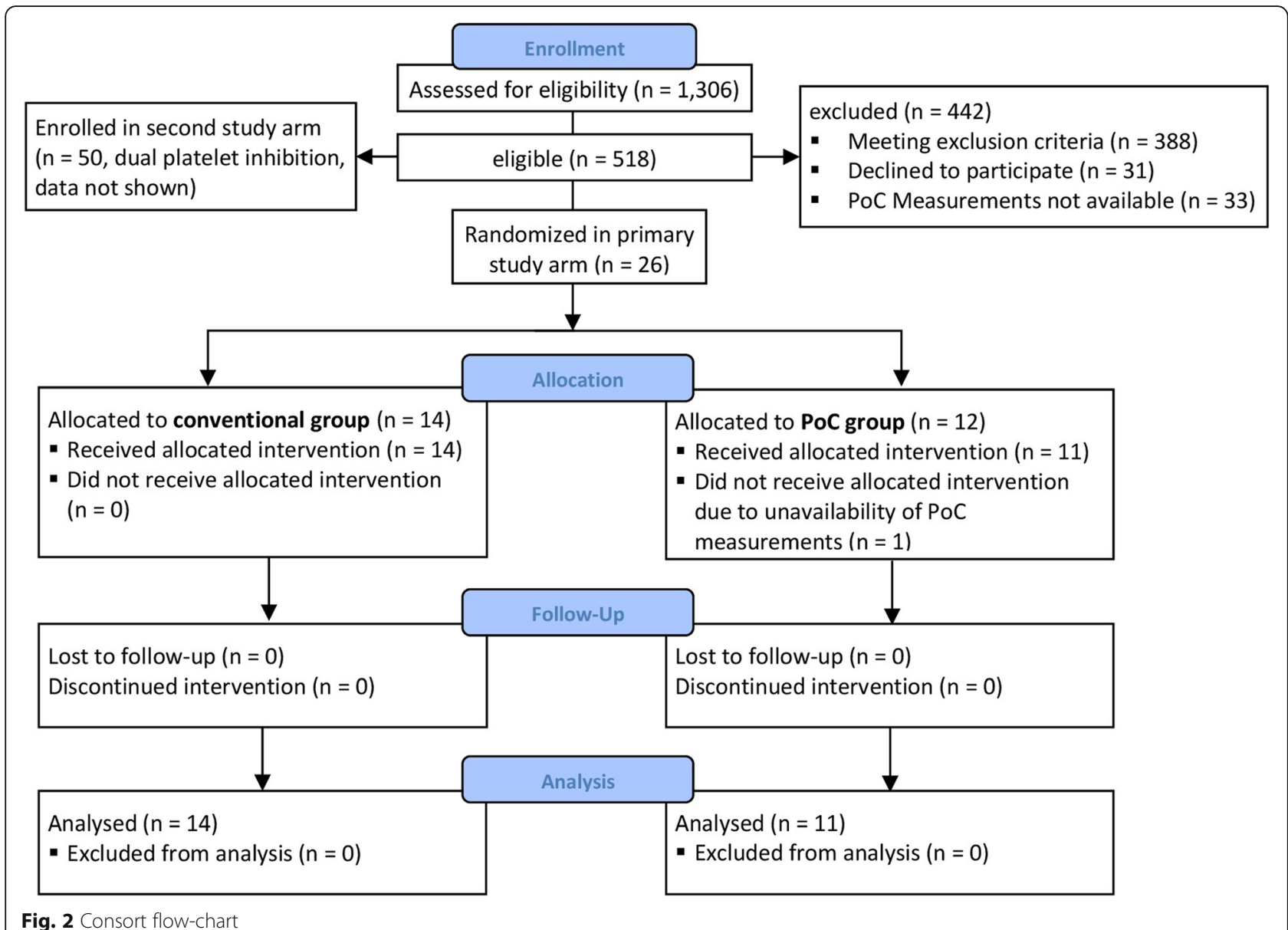

Table 1 Basic patient and surgery characteristics

\begin{tabular}{|c|c|c|c|}
\hline & $\begin{array}{l}\text { Conventional group } \\
(n=14)\end{array}$ & $\begin{array}{l}\text { PoC group } \\
(n=11)\end{array}$ & $P$ \\
\hline Sex [male] & $11(79)$ & $6(55)$ & 0.397 \\
\hline Age [years] & $70.5(65.5-74)$ & $71(69-76)$ & 0.501 \\
\hline $\mathrm{BSA}\left[\mathrm{m}^{2}\right]$ & $2.07(1.98-2,12)$ & $2.00(1.91-2.07)$ & 0.183 \\
\hline EuroScore & $5(4.5-6.3)$ & $6(6-8)$ & 0.166 \\
\hline $\mathrm{EF}[\%]$ & $60(54-62)$ & $57(50-73)$ & 0.893 \\
\hline Combined CABG and valve surgery & $10(71)$ & $7(64)$ & 1.000 \\
\hline Redo surgery & $4(29)$ & $3(27)$ & 1.000 \\
\hline Double valve surgery & $0(0)$ & $1(9)$ & 0.902 \\
\hline CPB time $[\mathrm{min}]$ & $107(87-129)$ & $115(99-135)$ & 0.291 \\
\hline Clamping time [min] & $80(59-95)$ & $98(69-120)$ & 0.344 \\
\hline Length of surgery [min] & $209(193-253)$ & $225(177-260)$ & 0.979 \\
\hline Length of surgical haemostasis [min] & $50(46-61)$ & $40(35-52)$ & 0.166 \\
\hline APACHE ॥ & $14.5(11-17.5)$ & $16(11-17)$ & 0.536 \\
\hline SAPS $\|$ & $28(25-32.25)$ & $31(25-35)$ & 0.403 \\
\hline Mechanical ventilation [hours] & $9(6-16)$ & $9(7-12)$ & 0.979 \\
\hline Incidents of RRT & 0 & $1(9.1)$ & 0.902 \\
\hline
\end{tabular}

Results are given as $\mathrm{n}(\%)$ or median (IQR). Differences were analysed using nonparametric Mann-Whitney $\mathrm{U}$ for or Chi-square test two independent samples with $a=0.05$ (two-sided)

Abbreviations: BSA Body surface area, EF Ejection fraction, CABG Coronary artery bypass grafting, CPB Cardio-pulmonary bypass, RRT Renal replacement therapy 


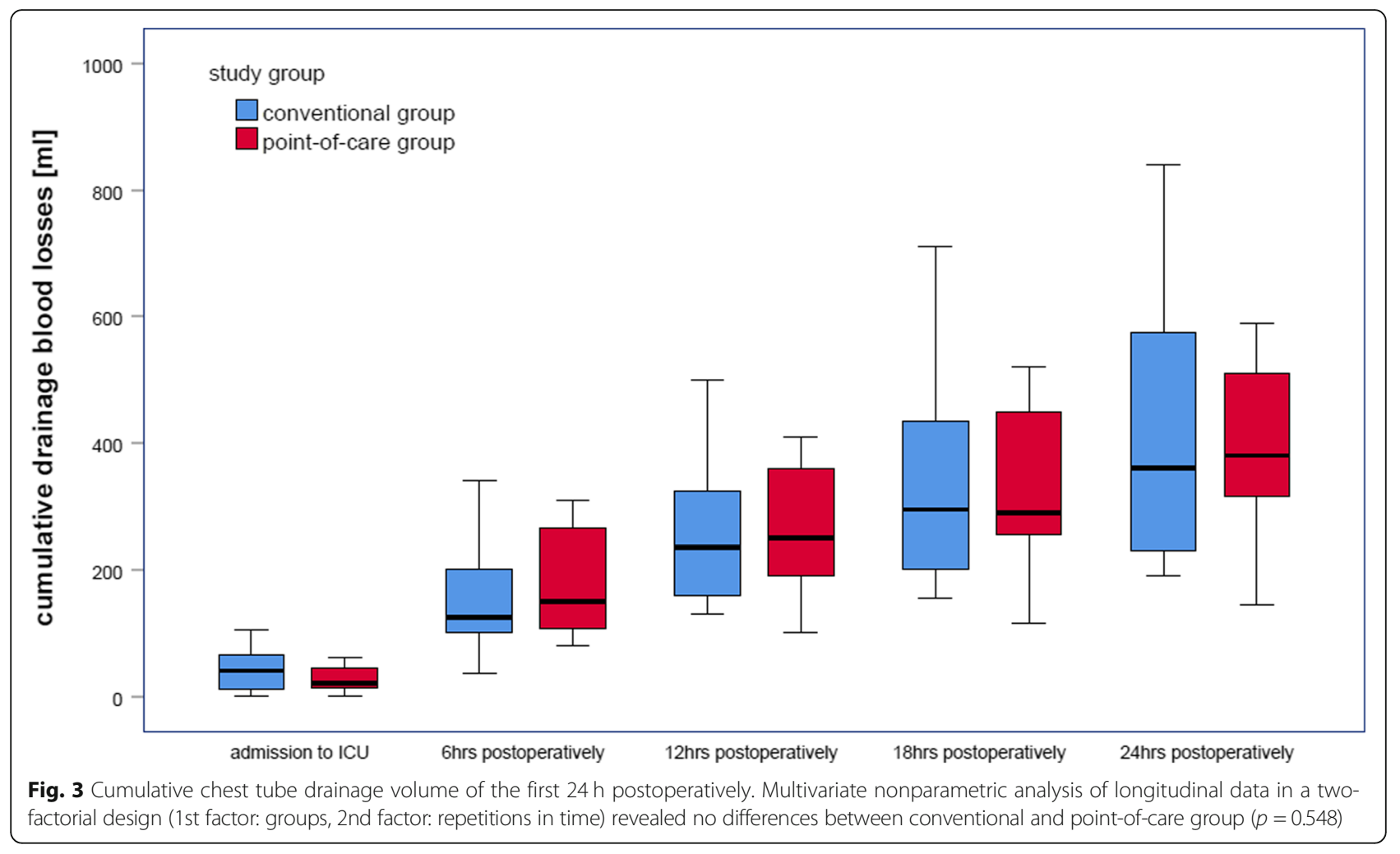

Table 2 Total transfusion rates or amounts of salvaged blood, RBCs, FFPs, platelets, fibrinogen, PPSB, and other haemostatic agents

\begin{tabular}{|c|c|c|c|}
\hline & $\begin{array}{l}\text { Conventional group } \\
(n=14)\end{array}$ & $\begin{array}{l}\text { PoC group } \\
(n=11)\end{array}$ & $P$ \\
\hline Retransfused, salvaged washed erythrocytes [ml] & $360(323-513)$ & $380(350-450)$ & 0.936 \\
\hline Total number of patients transfused with RBCs & $6(43 \%)$ & $8(72 \%)$ & 0.277 \\
\hline Thereof while on CPB & $3(21 \%)$ & $3(27 \%)$ & \\
\hline Thereof intraoperatively after CPB & $1(7 \%)$ & 0 & \\
\hline Thereof within $24 \mathrm{~h}$ postoperatively & $1(7 \%)$ & $4(36 \%)$ & \\
\hline Thereof within $48 \mathrm{~h}$ postoperatively & $4(29 \%)$ & $2(18 \%)$ & \\
\hline Later than $48 \mathrm{~h}$ postoperatively & $2(14 \%)$ & $5(45 \%)$ & \\
\hline Total number of patients transfused with platelets & 0 & $4(36 \%)$ & 0.056 \\
\hline Thereof intraoperatively after CPB & & $3(27 \%)$ & \\
\hline Thereof within $24 \mathrm{~h}$ postoperatively & & $2(18 \%)$ & \\
\hline Thereof within $48 \mathrm{~h}$ postoperatively & & 0 & \\
\hline Later than $48 \mathrm{~h}$ postoperatively & & 0 & \\
\hline Total number of PCC given & 0 & 0 & \\
\hline Total number of fibrinogen concentrate given (g) & 0 & 0 & \\
\hline Total number of patients transfused with FFP & $1(7 \%)$ & 0 & 1.000 \\
\hline Thereof intraoperatively after CPB & $1(7 \%)$ & & \\
\hline Others (desmopressin, protamine), total number of patients & 0 & 1 (9\%) (desmopressin) & \\
\hline
\end{tabular}


group only. The median number of platelet concentrates transfused was 2 (IQR 1.5, 2.5). Results of coagulation monitoring are given in Table 3. The only differences were a higher aPTT in the $\mathrm{PoC}$ group at $24 \mathrm{~h}$ postoperatively $(p=0.044)$, and a lower fibrinogen level in the PoC group at $6 \mathrm{~h}$ postoperatively $(p=0.006)$. Data of time point $6 \mathrm{~h}$ postoperatively is not included in Table 3 .

The secondary outcome parameters duration of mechanical ventilation postoperatively and the incidence of renal replacement therapy are also included in Table 1. Crystalloid/colloid infusions and urine output did not differ between the groups over the observation period (data not shown). Analyses were repeated after propensity matching. No significant differences regarding the impact of possible confounders were observed.

Protocol deviations occurred in three patients. The first case received $400 \mathrm{ml}$ of FFP in the conventional group in the initial phase of this study. The other two protocol deviations were the transfusion of two units of platelets at once, one also in the initial phase of the study. The second occurred intraoperatively prior to chest closure due to diffuse bleeding.

\section{Discussion}

A point-of-care guided transfusion algorithm did not result in less bleeding than a transfusion algorithm based on conventional coagulation test results in our study population. Transfusion requirements of RBCs and FFPs did not differ, while platelets were transfused in the PoC group only. There was no clinically significant difference in the course of coagulation parameters, duration of mechanical ventilation, or incidence of renal replacement therapy. Bleeding was less frequent and blood loss was lower than expected. Therefore, blood loss via chest tube drainage was not suitable to distinguish between a PoC- or central lab-guided transfusion algorithm. This may be attributed to the fact that surgery at high risk for perioperative bleeding may not sufficiently be defined only by the procedure but needs to include individual patient risk factors. In patients with insignificant bleeding point-of-care diagnostics do not seem to improve treatment and outcome as we detected no measurable difference. However, the low blood losses resulted in the early termination of this study.

Our results are in contrast to published studies showing a superiority of $\mathrm{PoC}$ guided algorithms in terms of reducing allogeneic blood transfusions [20, 23, 24]. Undoubtedly, transfusion algorithms - not covering RBCs are recommended to be used in severely bleeding patients only, as non-bleeding patients usually do not need any therapeutic intervention [16]. The relatively low blood losses postoperatively that usually do not require substitution with blood products may also explain why the haemostatic algorithm using PoC technique we used
Table 3 Course of coagulation parameters platelet count, aPTT, PT, fibrinogen, CT (Intem), CT (Extem), MCF (Fibtem), TRAP, ASPI, and ADP

\begin{tabular}{|c|c|c|c|}
\hline & $\begin{array}{l}\text { Conventional group } \\
(n=14)\end{array}$ & $\begin{array}{l}\text { PoC group } \\
(n=11)\end{array}$ & $P$ \\
\hline \multicolumn{4}{|l|}{ Platelet count [/nl] } \\
\hline Screening & $241(207-276)$ & $225(201-272)$ & 0.647 \\
\hline Admission to ICU & $153(111-184)$ & $150(120-191)$ & 0.893 \\
\hline $24 \mathrm{~h}$ postoperatively & $154(130-176)$ & $139(127-167)$ & 0.979 \\
\hline \multicolumn{4}{|l|}{$\mathrm{aPTT}[\mathrm{s}]$} \\
\hline Pre-operatively & $33.4(31.8-38.8)$ & $33.9(33.3-38.5)$ & 0.403 \\
\hline Admission to ICU & $35.2(33.4-37.3)$ & $38.1(37.3-40.9)$ & $0.038^{*}$ \\
\hline $24 \mathrm{~h}$ postoperatively & $34.6(32.4-37.9)$ & $38.1(34.5-43.3)$ & $0.044^{*}$ \\
\hline \multicolumn{4}{|l|}{ Thromboplastin time [\%] } \\
\hline Pre-operatively & $98(88-104)$ & $96(82-101)$ & 0.373 \\
\hline Admission to ICU & $57(55-65)$ & $60(54-62)$ & 0.851 \\
\hline $24 \mathrm{~h}$ postoperatively & $77(65-81)$ & $67(58-82)$ & 0.222 \\
\hline \multicolumn{4}{|l|}{ Fibrinogen [g/l] } \\
\hline Pre-operatively & $3.98(3.5-4.66)$ & $3.60(3.37-4.83)$ & 0.467 \\
\hline Admission to ICU & $2.58(2.17-3.42)$ & $2.48(2.09-3.07)$ & 0.699 \\
\hline $24 \mathrm{~h}$ postoperatively & $3.85(3.51-4.06)$ & $3.74(3.53-4.5)$ & 0.786 \\
\hline \multicolumn{4}{|l|}{ CT (Intem) [s] } \\
\hline Pre-operatively & $152(131-179)$ & $164(151-185)$ & 0.344 \\
\hline Admission to ICU & $188(179-201)$ & $195(177-213)$ & 0.536 \\
\hline $24 \mathrm{~h}$ postoperatively & $157(143-170)$ & $166(148-179)$ & 0.202 \\
\hline \multicolumn{4}{|l|}{$\mathrm{CT}$ (Extem) [s] } \\
\hline Pre-operatively & $52(48-57)$ & $60(51-62)$ & 0.149 \\
\hline Admission to ICU & $64(56-71)$ & $66(59-75)$ & 0.291 \\
\hline $24 \mathrm{~h}$ postoperatively & $55(48-63)$ & $53(46-64)$ & 0.767 \\
\hline \multicolumn{4}{|l|}{ MCF (Fibtem) [mm] } \\
\hline Pre-operatively & $23(21-25)$ & $22(19-24)$ & 0.572 \\
\hline Admission to ICU & $15(13-20)$ & $15(12-21)$ & 0.809 \\
\hline $24 \mathrm{~h}$ postoperatively & $22(20-25)$ & $24(20-28)$ & 0.424 \\
\hline \multicolumn{4}{|l|}{ TRAP [AU] } \\
\hline Pre-operatively & 119 (82-159) & $103(93-143)$ & 0.501 \\
\hline Admission to ICU & $139(93-159)$ & $116(85-149)$ & 0.572 \\
\hline $24 \mathrm{~h}$ postoperatively & $147(116-157)$ & $134(129-158)$ & 0.647 \\
\hline \multicolumn{4}{|l|}{ ASPI [AU] } \\
\hline Pre-operatively & $20(13-43)$ & $10(7-30)$ & 0.183 \\
\hline Admission to ICU & $20(12-48)$ & $24(5-52)$ & 0.893 \\
\hline $24 \mathrm{~h}$ postoperatively & $33(19-48)$ & $34(23-41)$ & 0.767 \\
\hline \multicolumn{4}{|l|}{$\mathrm{ADP}[\mathrm{AU}]$} \\
\hline Pre-operatively & $64(43-78)$ & $63(35-71)$ & 0.434 \\
\hline Admission to ICU & $61(44-72)$ & $45(33-82)$ & 0.476 \\
\hline $24 \mathrm{~h}$ postoperatively & $71(53-85)$ & 71 (58-90) & 0.727 \\
\hline
\end{tabular}

Results are given as median (IQR). Reference ranges of the local laboratory: Platelets 150-370/nl; aPTT 26-40s; thromboplastin time 70-130\%; fibrinogen $1.6-4.0 \mathrm{~g} / \mathrm{l}$. Reference ranges for activated rotational thromboelastometry: CT (Intem) 137-246 s; CT (Extem) 42-74 s; MCF (Fibtem) 9-25 mm. Reference ranges for multiple aggregometry: TRAP 84-128 AU; ASPI 71-115 AU; ADP 57$113 \mathrm{AU}$. Differences were analysed using nonparametric Mann-Whitney $\mathrm{U}$ test for two independent samples with $a=0.05$ (two-sided). Significant tests are marked with * 
for this study was not only not superior to an algorithm driven by conventional coagulation parameters, but may, in contrast, lead to a higher transfusion requirement when closely followed.

According to the EACTS/EACTA Guidelines on blood management, the first step towards creating algorithms is to identify patients at high risks for bleeding which are, following our results, not sufficiently defined by high-risk surgery only [33]. The anticipated chest tube drainage volume in our study which qualified patients for enrolment was unexpectedly low. We designed our study with the aim to compare patients undergoing surgery at high risk for bleeding without further risk factors for coagulopathy that might reduce comparability. This led to a sample of rather healthy subjects whose main risk for bleeding was the procedure itself. In this group, overall blood loss, incidence of relevant blood loss and transfusion requirements were remarkably below those of other studies [15, $21,23,24,34]$. This supports the idea that the definition of high-risk surgery should not only be made by the procedure itself but include individual patient risk factors, since better blood conservation technics have been established in the recent time [12, 35].

Nevertheless, there is a heterogeneity in the definition of bleeding. Our study enrolled patients preoperatively with a high risk for bleeding as predicted by the surgical procedure [3]. Bleeding was defined as intraoperative oozing delaying chest closure or as blood loss exceeding $1.5 \mathrm{ml} / \mathrm{kgBW} /$ hour in two consecutive hours or $4 \mathrm{ml} /$ $\mathrm{kgBW} /$ hour for at least $30 \mathrm{~min}$ postoperatively. The study of Weber et al., however, enrolled patients with "diffuse bleeding from capillary beds at wound surfaces requiring haemostatic therapy as assessed by the anaesthesiologist and surgeon by inspecting the operative field and/or intraoperative or postoperative blood loss exceeding $250 \mathrm{ml} / \mathrm{h}$ or $50 \mathrm{ml} / 10 \mathrm{~min}$ " [24]. $88 \%$ of their patients were enrolled intraoperatively without detailing which "coagulopathic" patients bled more than $250 \mathrm{ml} / \mathrm{h}$ or $50 \mathrm{ml} / 10 \mathrm{~min}$ or were bleeding diffusely. This inclusion criterion might be less precise compared to another method described, i.e. quantifying bleeding intraoperatively by packing with and weighing of sponges [36].

Furthermore, Weber et al. enrolled 100 of 152 eligible patients intraoperatively [24]. A larger sample of 1144 patients assessed according to the universal definition of perioperative bleeding found a distribution of $51.4 \%$ patients experiencing insignificant bleeding only, mild bleeding in 14.9\% or moderate bleeding in $24 \%$ of patients, while severe or massive bleeding occurred in $9.8 \%$ of the patients [6].

Our study included 25 patients with a high risk of bleeding, of whom $20 \%$ experienced a blood loss requiring transfusion of haemostatic blood products excluding RBCs. The majority of patients suffered insignificant bleeding; blood losses were much lower than in previously published studies [15, 21, 23, 24, 34], that may explain the different results. There are several potential reasons for these differences: advances in surgical technique, implementation of patient blood management programs and even more important a more meticulous haemostasis by cardiac surgeons who were not blinded to the study group allocation.

For the secondary outcome parameter, transfusion requirements, our results are also contrary to earlier work [20]. This might be due to the lower incidence of bleeding in general. However, despite the small sample, the incidence of platelet transfusions in the PoC group might require more attention, as $36 \%$ of the patients were transfused compared to none in the conventional group. The decision to transfuse platelet concentrates should not be based on lab testing alone, but also on the clinical condition of the patient and the amount of blood loss. This aspect should especially be considered when facing patients that are rated as at high risk for bleeding, therefore being examined with $\mathrm{PoC}$ parameters but finally not having significant blood losses. Future clinical studies addressing PoC-based transfusion triggers for platelets in bleeding patients might help shedding light on this issue.

This study has some limitations: First, the limited number of included patients results in lack of power [37]. Second, we recognized three protocol deviations accounting for $12 \%$ of patients. This may have caused some bias influencing the results [38]. However, $88 \%$ were treated according to the treatment protocol, which is a comparably high rate of protocol adherence. Third, blinding was not possible in this study as PoC coagulation testing started in the operation theatre, which may have influenced surgeons to perform a better surgical haemostasis in all patients. Furthermore, due to the hospital information system, blinding of the attending physicians to the conventional coagulation parameters was not possible, although the transfusion protocol did not allow for transfusions based on those parameters in the PoC group. Fourth, criteria to exclude patients with haemostasis defects were strict, causing a low overall percentage of eligibility. This might reduce the generalizability of this study. Last, our definition of bleeding risk by surgical procedure did not include all patients with a high risk of bleeding, e.g. patients on dual antiplatelet therapy, in whom platelet function testing may have yielded better results than a platelet count driven transfusion algorithm. However, the type of surgery remains an important factor to define the risk of bleeding in cardiac surgery $[21,24,25]$.

\section{Conclusion}

This study suggests that blood losses and transfusion amounts did not differ comparing PoC- and central lab-driven transfusion algorithms in high-risk cardiac surgery, when drainage losses are small $(<500 \mathrm{ml} / 12 \mathrm{~h})$ 
and surgeons not blinded to study group allocation. The definition of high-risk for bleeding should possibly include individual risk factors rather than procedural factors only. Physicians should take these limitations into account when considering $\mathrm{PoC}$ measurements for this patient group. These results need re-evaluation in a larger prospective and randomized multicentre trial implementing explicit and restrictive goal-directed transfusion algorithms in both groups.

\section{Appendix}

Exclusion Criteria:

- age $<18$ or $>80$ years

- known haemophilia

- known thrombophilia

- known hereditary thrombocytopathy

- hereditary or acquired coagulation disorder (not

including single antiplatelet therapy)

- active endocarditis

- ejection fraction $<30 \%$

- $\mathrm{BSA}<1.8 \mathrm{sqm}$

- planned aortic arch surgery

- preoperative thrombocytopenia $<150 / \mathrm{nl}$

- underlying haemostaseological disease

- preoperative anaemia

- liver cirrhosis Child B or higher

- preoperative creatinine $>2 \mathrm{mg} / \mathrm{dl}$

- terminal renal insufficiency requiring dialysis

- vitamin $\mathrm{k}$ antagonists during 5 days prior to surgery

- pregnant or breastfeeding women

- known allergy against allogeneic blood products or

coagulation factors

- refusal of blood transfusions

- any concomitant investigational agent or participation

in another trial

\section{Abbreviations}

ACT: Activated clotting time; aPTT: Activated Partial Thromboplastin Time; BIS: Bispectral index; CABG: Coronary artery bypass graft; CPB: Cardiopulmonary bypass; FFP: Fresh frozen plasma; IQR: Interquartile ranges; MEA: Multiple electrode aggregometry; PoC: Point-of-care; RBC: Packed red blood cells; ROTEM: Rotational thromboelastometry; TRALI: Transfusion associated lung injury

\section{Acknowledgements}

Not applicable.

\section{Funding}

This study was funded by institutional grants of Charité - Universitätsmedizin Berlin only. These were used for point-of-care materials and central lab parameter calculation as well as cellsaver usage. The funding body did neither influence the collection of data nor the interpretation of results.

\section{Availability of data and materials}

The datasets used and/or analyzed during the current study are available from the corresponding author on reasonable request. The complete trial protocol for this investigator-initiated, single-center trial is available in German only, and can be requested by the corresponding author.

\section{Authors' contributions}

FL: Study design, analysis of data, patient recruitment, data collection and drafting the manuscript. JR: Study design, analysis of data, drafting and critically revising the manuscript. LK, MS and $\mathrm{CVH}$ : Study design, analysis of data, and critically revising the manuscript. BM, TM, and TG: Patient recruitment, data collection, and critical revision of the manuscript. KL: critical revision of the manuscript. KDW and FB: statistical analysis and critical revision of the manuscript. All authors have approved the final version of the manuscript.

\section{Ethics approval and consent to participate}

The study was conducted at the Department of Anaesthesiology and Intensive Care Medicine of Charité - University Medicine Berlin, Berlin, Germany in adherence to the latest version of the declaration of Helsinki, approved by the local Ethics Board (Ethics Committee of Charité - University Medicine Berlin (EA1/263/10). All patients gave written informed consent prior to entering the study.

\section{Consent for publication \\ Not applicable.}

\section{Competing interests}

$J R, B M, T M$, and TG have no conflicts of interest. KDW received honoraria from Charité - University Medicine Berlin related to the topic of this study. LK received honoraria for lectures related to the topic of this work as well as travel reimbursements from CSL Behring, NovoNordisk GmbH, TEM International, Sinetica and HICC GbR. CvH received honoraria for lectures and consultancy work related to the topic of this work as well as travel reimbursements from CSL Behring, Ferring GmbH, NovoNordisk GmbH, Haemonetics and HICC GbR. FL received travel reimbursements from Edwards Lifesciences. FB received grants, personal fees and/or non-financial supports from Einstein Foundation, Axon Publishing, Clearflow, Vifor Pharma and Medtronic. MS received grants from Medtronic, Edwards Lifesciences, AMOMED, Fisher\&Peykel, Grünenthal, Massimo and Gettinge Group.

\section{Publisher's Note}

Springer Nature remains neutral with regard to jurisdictional claims in published maps and institutional affiliations.

\section{Author details}

'Department of Anaesthesiology and Intensive Care Medicine, Charité Universitätsmedizin Berlin, Campus Virchow-Klinikum and Campus Charité Mitte, Berlin, Germany. ${ }^{2}$ Division of Medical Biotechnology, Paul-Ehrlich-Institut, Federal Institute for Vaccines and Biomedicines, Langen, Hessen, Germany. ${ }^{3}$ Department of Internal Medicine, Hegau-Bodensee-Klinikum, Singen, Baden-Württemberg, Germany. ${ }^{4}$ Department of Anaesthesiology, Intensive Care Medicine and Pain therapy, University Hospital Gießen UKGM, Justus-Liebig-University Giessen, Giessen, Hessen, Germany. ${ }^{5}$ Department of Anaesthesia, Intensive Care Medicine, Emergency Medicine and Pain Therapy, Vivantes Klinikum im Friedrichshain, Berlin, Germany. ${ }^{6}$ Department of Anaesthesiology and Intensive Care Medicine, Martin-Luther-Krankenhaus, Berlin, Germany. ${ }^{7}$ CRO SOSTANA $\mathrm{GmbH}$ and Charité - Universitätsmedizin Berlin, Berlin, Germany.

Received: 19 November 2018 Accepted: 25 January 2019

Published online: 18 February 2019

\section{References}

1. Miceli A, et al. Combined clopidogrel and aspirin treatment up to surgery increases the risk of postoperative myocardial infarction, blood loss and reoperation for bleeding in patients undergoing coronary artery bypass grafting. Eur J Cardiothorac Surg. 2013;43(4):722-8.

2. Herman $C R$, et al. Clopidogrel increases blood transfusion and hemorrhagic complications in patients undergoing cardiac surgery. Ann Thorac Surg. 2010;89(2):397-402.

3. Hardy JF, et al. The stratification of cardiac surgical procedures according to use of blood products: a retrospective analysis of 1480 cases. Can J Anaesth. 1991:38(4 Pt 1):511-7.

4. Levi M, et al. Pharmacological strategies to decrease excessive blood loss in cardiac surgery: a meta-analysis of clinically relevant endpoints. Lancet. 1999;354(9194):1940-7. 
5. Christensen MC, et al. Increased chest tube drainage is independently associated with adverse outcome after cardiac surgery. J Cardiothorac Vasc Anesth. 2012;26(1):46-51.

6. LaPar DJ, et al. Blood product conservation is associated with improved outcomes and reduced costs after cardiac surgery. J Thorac Cardiovasc Surg. 2013;145(3):796-803 discussion 803-4.

7. Shander A, et al. Patient blood management in Europe. Br J Anaesth. 2012; 109(1):55-68.

8. Bisbe E, Molto L. Pillar 2: minimising bleeding and blood loss. Best Pract Res Clin Anaesthesiol. 2013:27(1):99-110.

9. Goodnough LT, Shander A. Patient blood management. Anesthesiology. 2012;116(6):1367-76.

10. Song HK, et al. Safe application of a restrictive transfusion protocol in moderate-risk patients undergoing cardiac operations. Ann Thorac Surg. 2014;97(5):1630-5.

11. Moskowitz DM, et al. The impact of blood conservation on outcomes in cardiac surgery: is it safe and effective? Ann Thorac Surg. 2010;90(2):451-8.

12. Society of Thoracic Surgeons Blood Conservation Guideline Task Force, et al. 2011 update to the society of thoracic surgeons and the society of cardiovascular anesthesiologists blood conservation clinical practice guidelines. Ann Thorac Surg. 2011;91(3):944-82.

13. Kozek-Langenecker SA, et al. Management of severe perioperative bleeding: guidelines from the European society of anaesthesiology: first update 2016. Eur J Anaesthesiol. 2017;34(6):332-95.

14. Görlinger K, Dirkmann D, Hanke AA. Potential value of transfusion protocols in cardiac surgery. Curr Opin Anaesthesiol. 2013;26(2):230-43.

15. Royston D, von Kier S. Reduced haemostatic factor transfusion using heparinase-modified thrombelastography during cardiopulmonary bypass. Br J Anaesth. 2001;86(4):575-8.

16. Theusinger OM, Stein P, Levy JH. Point of care and factor concentrate-based coagulation algorithms. Transfus Med Hemother. 2015;42(2):115-21.

17. Ferraris VA, et al. 2012 update to the society of thoracic surgeons guideline on use of antiplatelet drugs in patients having cardiac and noncardiac operations. Ann Thorac Surg. 2012;94(5):1761-81.

18. Rossaint $R$, et al. The European guideline on management of major bleeding and coagulopathy following trauma: fourth edition. Crit Care. 2016;20:100

19. (NICE), N.I.f.H.a.C.E, Detecting, managing and monitoring haemostasis: viscoelastometric point-of-care testing (ROTEM, TEG and Sonoclot systems). 2014.

20. Wikkelso A, et al. Thromboelastography (TEG) or thromboelastometry (ROTEM) to monitor haemostatic treatment versus usual care in adults or children with bleeding. Cochrane Database Syst Rev. 2016;8:CD007871 https://doi.org/10.1002/14651858.CD007871.pub3.

21. Ak K, et al. Thromboelastography-based transfusion algorithm reduces blood product use after elective CABG: a prospective randomized study. J Card Surg. 2009;24(4):404-10.

22. Nuttall GA, et al. Efficacy of a simple intraoperative transfusion algorithm for nonerythrocyte component utilization after cardiopulmonary bypass. Anesthesiology. 2001;94(5):773-81 discussion 5A-6A.

23. Shore-Lesserson $\mathrm{L}$, et al. Thromboelastography-guided transfusion algorithm reduces transfusions in complex cardiac surgery. Anesth Analg. 1999;88(2): 312-9.

24. Weber CF, et al. Point-of-care testing: a prospective, randomized clinical trial of efficacy in coagulopathic cardiac surgery patients. Anesthesiology. 2012; 117(3):531-47

25. Westbrook AJ, et al. Protocol based on thromboelastograph (TEG) outperforms physician preference using laboratory coagulation tests to guide blood replacement during and after cardiac surgery: a pilot study. Heart Lung Circ. 2009;18(4):277-88.

26. Manikappa $\mathrm{S}$, et al. Changes in transfusion therapy guided by thromboelastograph in cardiac surgery. Ann Card Anaesth. 2001;4(1):21-7.

27. Gawande A. The checklist manifesto : how to get things right. 1st ed. New York: Metropolitan Books; 2010. p. 209.

28. Fergusson DA, et al. A comparison of aprotinin and lysine analogues in high-risk cardiac surgery. N Engl J Med. 2008;358(22):2319-31.

29. Everts PA, et al. Is the use of autologous platelet-rich plasma gels in gynecologic, cardiac, and general, reconstructive surgery beneficial? Curr Pharm Biotechnol. 2012;13(7):1163-72.

30. Toth $\mathrm{O}$, et al. Multiple electrode aggregometry: a new device to measure platelet aggregation in whole blood. Thromb Haemost. 2006;96(6):781-8.
31. Diamond A, Sekhon JS. Genetic matching for estimating causal effects: a general multivariate matching method for achieving balance in observational studies. Rev Econ Stat. 2013;95(3):932-45.

32. Sekhon JS. Multivariate and Propensity Score Matching Software with Automated Balance Optimization: The Matching package for R. Journal of Statistical Software. 2011:42:1-52.

33. Pagano D, et al. 2017 EACTS/EACTA Guidelines on patient blood management for adult cardiac surgery. Eur J Cardiothorac Surg. 2018;53(1): 79-111.

34. Avidan MS, et al. Comparison of structured use of routine laboratory tests or near-patient assessment with clinical judgement in the management of bleeding after cardiac surgery. Br J Anaesth. 2004;92(2):178-86.

35. European Society of Anaesthesiology task force reports on place of aprotinin in clinical anaesthesia. Aprotinin: is it time to reconsider? Eur J Anaesthesiol. 2015:32(9):591-5.

36. Karkouti $\mathrm{K}$, et al. Evaluation of a novel transfusion algorithm employing point-of-care coagulation assays in cardiac surgery: a retrospective cohort study with interrupted time-series analysis. Anesthesiology. 2015;122(3): 560-70.

37. Spahn DR. TEG(R)- or ROTEM(R)-based individualized goal-directed coagulation algorithms: don't wait--act now! Crit Care. 2014;18(6):637.

38. Rahe-Meyer N, et al. Randomized evaluation of fibrinogen vs placebo in complex cardiovascular surgery (REPLACE): a double-blind phase III study of haemostatic therapy. Br J Anaesth. 2016;117(1):41-51.
Ready to submit your research? Choose BMC and benefit from:

- fast, convenient online submission

- thorough peer review by experienced researchers in your field

- rapid publication on acceptance

- support for research data, including large and complex data types

- gold Open Access which fosters wider collaboration and increased citations

- maximum visibility for your research: over $100 \mathrm{M}$ website views per year

At BMC, research is always in progress.

Learn more biomedcentral.com/submissions 